\title{
Left atrial Intramural hematoma after radiofrequency catheter ablation of left lateral accessory pathway
}

\author{
Salim Yaşar * (D, Yalçın Gökoğlan, Suat Görmel, Serkan Asil and Hasan Kutsi Kabul
}

\begin{abstract}
We report a case of left atrial hematoma after ablation of left lateral concealed accessory pathway. A 46-year-old male patient experienced chest pain after radiofrequency ablation. Transthoracic echocardiography and computed tomography revealed the intramural mass consistent with hematoma in the left atrium. He was hemodynamically stable, and conservative approach was decided. Atrioventricular groove is a vulnerable part of left atrium, and ablation of left free wall accessory pathway may require targeting both atrial and ventricular surfaces of the mitral annulus. Avoidance of forceful catheter manipulation during the electrophysiological procedure is important for prevention of this complication. Optimal periprocedural anticoagulation might reduce the risk of procedure-related thromboembolic complications, but electrophysiologists should always pay attention to an intramural hematoma that may occur after radiofrequency catheter ablation.
\end{abstract}

Keywords: Supraventricular tachycardia, Radiofrequency catheter ablation, Hematoma

\section{Case report}

A 46 year-old man presented to our clinic with a complaint of palpitation. Electrocardiogram during one of these palpitation episodes showed narrow complex tachycardia with a rate of $190 / \mathrm{min}$. He reported that his palpitation continued despite various antiarrhythmic (metoprolol and propafenon) treatments. His past medical history was unremarkable. Baseline electrocardiogram was in normal limits without any preexcitation. All laboratory values and a baseline transthoracic echocardiography (TTE) were normal. Treatment options were discussed, and electrophysiological study/catheter ablation was scheduled. At electrophysiological study, orthodromic atrioventricular reentrant tachycardia using left lateral concealed accessory pathway was induced. Intravenous heparin

*Correspondence: dr.salimyasar@hotmail.com Department of Cardiology, Gülhane Training and Research Hospital, 06010 Ankara, Turkey
$(100 \mathrm{U} / \mathrm{kg})$ was administered after femoral arterial sheath insertion. Radiofrequency (RF) ablation was performed via retrograde aortic approach without any difficulties (Fig. 1). Ablation was carried out with Medtronic Atakr RF generator and single curve, tipdeflecting bipolar, non-irrigated catheter (Medtronic RF Marinr) with temperature limited to $55^{\circ} \mathrm{C}$ and power limited to 40 Watt. After ablation, there was no AP conduction and tachycardia was not induced by programmed stimulation with or without isoproterenol infusion. The morning after the ablation, patient was feeling well and he was discharged with acetylsalicylic acid $100 \mathrm{mg}$. Two days following ablation, patient was admitted with chest pain lasting for $12 \mathrm{~h}$. Electrocardiogram was unremarkable. TTE revealed a well-circumscribed hypoechogenic mass in the lateral wall of the left atrium, suggestive of an intramural hematoma (Fig. 2). There was no evidence of left ventricular inflow obstruction. He was hemodynamically stable. Then, the patient underwent contrast-enhanced cardiac 


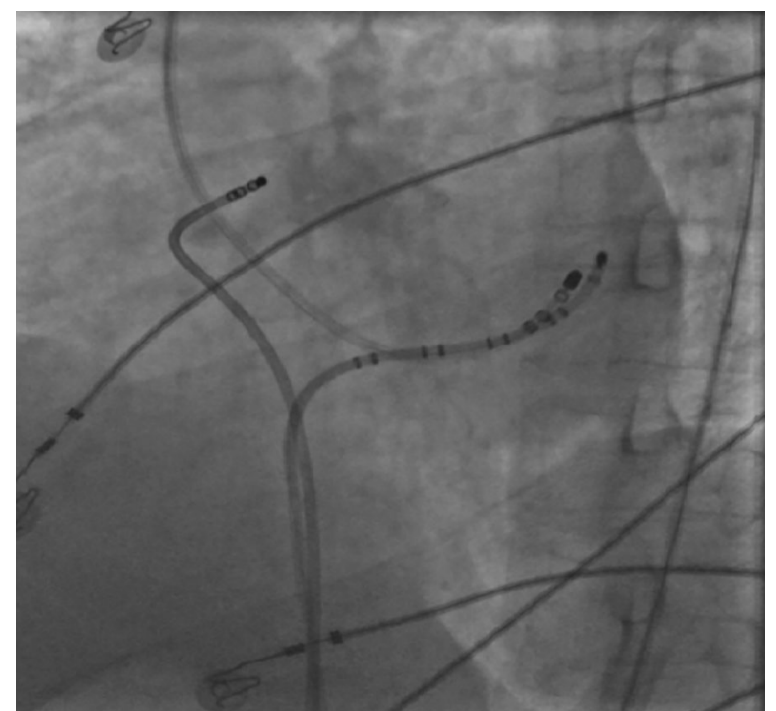

Fig. 1 Left lateral view of catheter positions during ablation

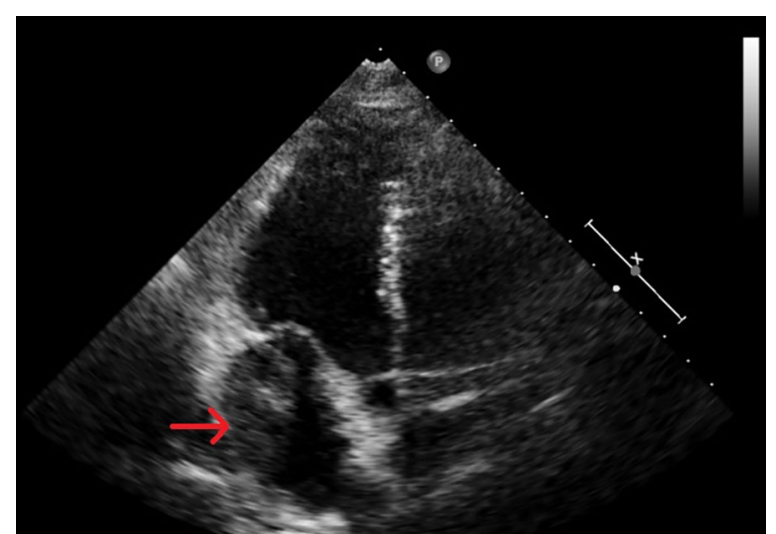

Fig. 2 Transthoracic echocardiogram in apical four-chamber view shows well-circumscribed hypoechogenic mass in the lateral wall of the left atrium computed tomography $(\mathrm{CT})$ to investigate the nature of the mass which revealed homogeneous intramural mass consistent with $30-\times 19-\mathrm{mm}$ hematoma located in the left lateral wall (Fig. 3,4). So imaging revealed left atrial intramural hematoma (LAIH). There was no pulmonary vein obstruction. Since there was no hemodynamic instability, watchful monitoring was decided. Acetylsalicylic acid was continued, and analgesics were administered if needed. Follow-up CT 3 days after the index scan revealed partial regression of hematoma. The patient remained well clinically and was discharged from the hospital at the sixth day of hospitalization without any symptoms. A control TTE 2 months later

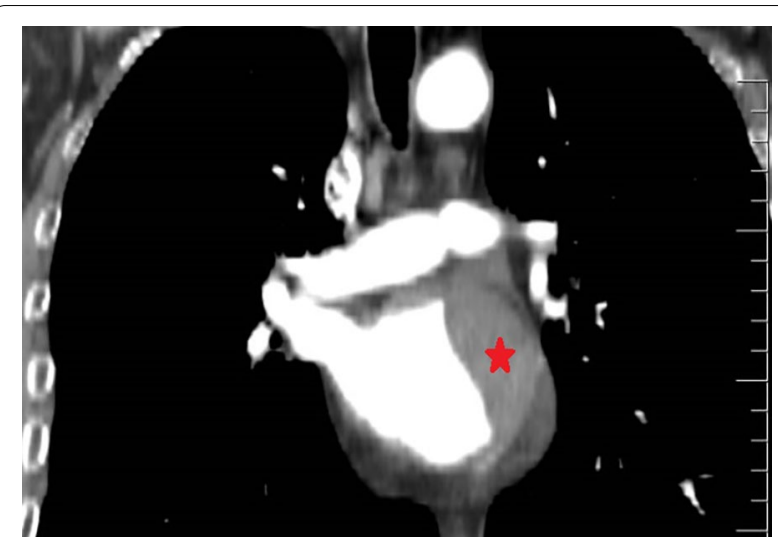

Fig. 3 Coronal computed tomography (CT) imaging demonstrating a non-enhancing left atrial intramural mass consistent with hematoma

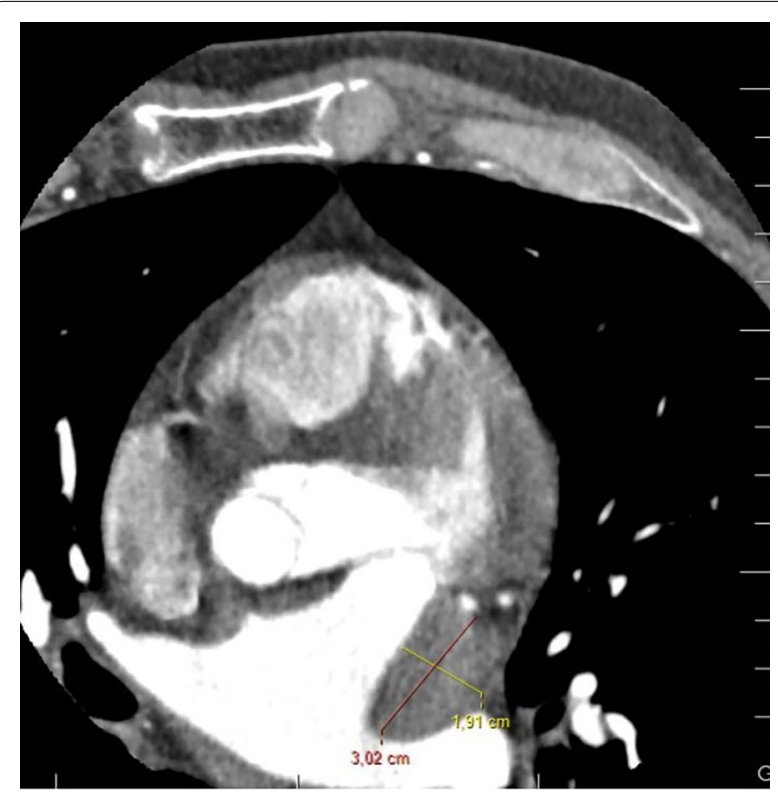

Fig. 4 Axial computed tomography (CT) imaging demonstrating a non-enhancing left atrial intramural mass consistent with hematoma

showed complete resolution of intramural hematoma (Fig. 5).

\section{Discussion}

RF catheter ablation of atrial tachyarrhythmias may lead to complications such as thromboembolism and bleeding [1]. LAIH are extremely rare in this context. LAIH is an extremely rare complication of cardiac surgical or catheter-based interventional procedures. Cases of LAIH have been reported as a complication of RF ablation procedures for the treatment of left atrial arrhythmias, including atrial fibrillation, atrial tachycardias [2, 3]. Myung-jin 


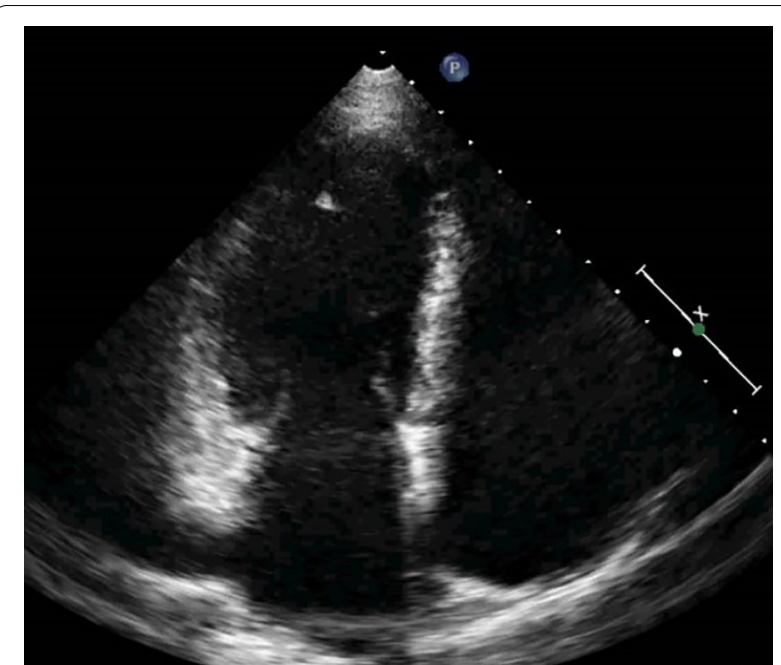

Fig. 5 Control transthoracic echocardiogram in apical four-chamber view shows complete resolution of intramural hematoma

Cha and Seil Oh reported a case of patient with an intramural hematoma associated with RFCA for intractable atrial tachyarrhythmias, who was treated with surgery [4]. The first case of LAIH as a complication of left posterolateral concealed accessory pathway ablation using retrograde aortic approach reported by Giudicatti et al. [5]. Similarly, our case has a LAIH as a complication of left lateral concealed accessory pathway RF ablation too. True incidence and pathophysiology of LAIH are poorly understood. Thermal injury and catheter manipulation may entail intimal tear in vulnerable portions of left atrium [6, 7]. Atrioventricular groove also is a naturally weakened transitional area vulnerable to forceful manipulation [8]. We conclude that both catheter manipulation and thermal injury account for occurrence of LAIH in our case. Avoidance of forceful catheter manipulation, especially in left atrium, is important for prevention of this complication.

\section{Conclusion}

The clinical course of LAIH varies from a self-limited stable disease to a devastating condition with catastrophic outcomes. Hemodynamic instability is the critical factor that determines the necessity of surgical intervention, and it is mainly secondary to obstruction of mitral valve inflow or pulmonary vein orifice. In case of hemodynamic instability, surgical intervention is indicated. However, patients with stable hemodynamic status, as was seen in our patient, can be observed clinically.

\section{Abbreviations}

TTE: Transthoracic echocardiography; RF: Radiofrequency; CT: Computed tomography; LAlH: Left atrial intramural hematoma.

\section{Acknowledgements}

Not applicable.

\section{Authors' contributions}

Each author contributed equally. All authors read and approved the final manuscript.

\section{Funding}

This case report received no specific grant from any funding agency in the public, commercial, or not-for-profit sectors.

\section{Availability of data material}

Since it is a single case report, only clinical records were available.

\section{Declarations}

Ethics approval and consent to participate

Single case report. Not applicable.

\section{Consent for publication}

Written informed consent for publication of their clinical details and/or clinical images was obtained from the patient.

\section{Competing interests}

The authors declare no conflict of interests for this article.

Received: 13 May 2021 Accepted: 11 June 2021

Published online: 19 July 2021

References

1. Zhou L, Keane D, Reed G, Ruskin J. Thromboembolic complications of cardiac radiofrequency catheter ablation: a review of the reported incidence, pathogenesis and current research directions. J Cardiovasc Electrophysiol. 1999;10:611-20.

2. Sah R, Epstein LM, Kwong RY. Images in cardiovascular medicine. Intramural atrial hematoma after catheter ablation for atrial tachyarrhythmias. Circulation. 2007;115:e446-447.

3. Kelly S, Bicknell SG, Sharma S. Left atrial wall hematoma after radiofrequency ablation for atrial fibrillation. AJR Am J Roentgenol. 2006;186:1317-9.

4. Cha MJ, Oh S. Left atrial Intramural hematoma after catheter ablation for atrial tachyarrhythmia. Arrhythmia. 2015;16(2):117-20.

5. Giudicatti L, King B, Lee F, Lam K, Stamp N, Gupta A, McClelland S, Xu $Z$-F. Left atrial intramural hematoma post-ablation of supraventricular tachycardia: a rare complication treated successfully. J Am Coll Cardiol Case Rep. 2020;2(2):223-6.

6. Kurek C, Gwechenberger M, Richter B, Binder T, Loewe C, Gössinger H. Intramural left atrial haematoma mimicking cardiac tamponade after catheter ablation of atria fibrillation. Europace. 2009;11:667-8.

7. Morris MF, Adams JC, Panse PM, Srivathsan K. Radiofrequency ablation complicated by left atrial intramural hematoma. J Cardiovasc Electrophysiol. 2012;23:662-3.

8. Lawton JS, Deshpande SP, Zanaboni PB, Damiano RJ Jr. Spontaneous atrioventricular groove disruption during off-pump coronary artery bypass grafting. Ann Thorac Surg. 2005;79:339-41. 